THE Astrophysical JouRnAL, 547:82-89, 2001 January 20

(C) 2001. The American Astronomical Society. All rights reserved. Printed in U.S.A.

\title{
RADIAL DENSITY AND TEMPERATURE PROFILES OF THE INTRACLUSTER GAS CONSTRUCTED JOINTLY FROM THE X-RAY SURFACE BRIGHTNESS MEASUREMENT AND THE UNIVERSAL DENSITY PROFILE
}

\author{
XIANG-PING Wu \\ Beijing Astronomical Observatory and National Astronomical Observatories, Chinese Academy of Sciences, Beijing 100012, China \\ AND \\ TZIHONG CHIUEH \\ Department of Physics, National Taiwan University, Taipei 10746; and Institute of Astronomy and Astrophysics, Academia Sinica, Taipei, Taiwan 115, \\ Republic of China \\ Received 2000 March 26; accepted 2000 September 27
}

\begin{abstract}
In this paper we have made an attempt to derive the radial profiles of the density and temperature of intracluster gas based on two currently well-established facts: the X-ray observed surface brightness of clusters described by the standard $\beta$ model, and the (NFW) universal density profile as the underlying dark matter distribution. We have numerically solved the hydrostatic equation by requiring that the volume-averaged baryon fraction of a cluster should asymptotically approach the universal value at its viral radius. We have shown that the radial temperature variation derived from these constraints differs significantly from the conventional polytropic equation of state: the gas temperature profile may show a dramatic increase or decrease with outward radius, depending sensitively on the $\beta$ parameter. A large $\beta$ value (typically $>0.8$ ) is required in order to ensure that the X-ray temperature makes a drop at the virial radius. This indicates either that the NFW profile is inappropriate to apply to the overall dark matter distribution of clusters, or that other nongravitational heating processes may play an important role in the dynamical evolution of clusters.
\end{abstract}

Subject headings: cosmology: theory — dark matter — galaxies: clusters: general intergalactic medium - X-rays: galaxies

\section{INTRODUCTION}

One of the major uncertainties in the cosmological application of the dynamical properties and evolution of galaxy clusters, e.g., the baryon fraction $f_{b}$ and the X-ray luminosity-temperature correlation, has probably arisen from the poorly constrained radial variations of the density, and especially the temperature, of hot intracluster gas. Indeed, the currently available X-ray spectral data of clusters in the literature may have suffered various instrumental limitations (see, e.g., Irwin, Bregman, \& Evrard 1999), and even conflicting results regarding the radial temperature gradients have been obtained by different authors based on different analysis techniques (e.g., Markevitch et al. 1998; Irwin et al. 1999; White 2000; Irwin \& Bregman 2000). This leads to an embarrassing situation in which an oversimplified, isothermal gas distribution is often adopted in the estimate of the total gravitating mass, $M_{\mathrm{tot}}(r)$, of a cluster enclosed within radius $r$ via the hydrostatic equilibrium hypothesis. Here it is worth mentioning another possibility for obtaining the radial distribution of intracluster gas in an indirect way, namely, by the inversion of the X-ray and Sunyaev-Zeldovich (SZ) observed surface brightness profiles of clusters (Silk \& White 1978; Yoshikawa \& Suto 1999). However, the actual application of this technique to clusters is still limited, primarily by the current SZ measurements.

Several efforts have thus been made toward deriving the radial profiles of the density and temperature of intracluster gas based on well-motivated physical mechanisms. Among these, the shape of the gas density distribution following the universal density profile of the dark matter halos of clusters suggested by numerical simulations (Navarro, Frenk, \&
White 1995, hereafter NFW) has generated great interest (e.g., Makino, Sasaki, \& Suto 1998; Ettori \& Fabian 1999; Wu \& Xue 2000a; Wu 2000; etc.). While its inner and outer slopes are still under debate, the NFW profile can be considered to be a good approximation of the matter distribution of dark halos. Alternatively, a number of highresolution simulations have also shown that the NFW profile is independent of mass, the initial density fluctuation, and the cosmology (Cole \& Lacey 1996; Navarro et al. 1997; Eke, Navarro, \& Frenk 1998). Indeed, within the framework of isothermal and hydrostatic equilibrium, there is a striking similarity between the gas density distribution predicted by the NFW profile and the conventional $\beta$ model revealed by the X-ray observations (Makino et al. 1998). Inclusion of the self-gravity of the gas and the polytropic equation of state makes it promising to directly link the theoretical predictions with the actual X-ray observations of clusters (Suto, Sasaki, \& Makino 1998).

Here we view the problem from a different angle: given (1) the radial variation of the dark matter particles characterized by the NFW profile and (2) the X-ray observed surface brightness profiles of clusters, what can we learn about the radial density and temperature profiles of the intracluster gas? Namely, we begin with the two wellestablished "observational" facts, and drop the assumption about the temperature properties of the gas (isothermal or polytropic). Nevertheless, we include the self-gravity of the gas. We then solve the hydrostatic equation with the following boundary constraint: the volume-averaged baryon fraction $f_{b}(r)$ within the virial radius $r_{\text {vir }}$ should asymptotically approach a universal value $f_{b \text {, BBN }} \equiv \Omega_{b} / \Omega_{m}$ defined by big bang nucleosynthesis, where $\Omega_{b}$ and $\Omega_{m}$ are the average 
baryon and total mass densities of the universe, respectively, in units of the critical density $\rho_{\text {crit }}$ for closure. The reasons for imposing this condition on the solution are as follows. First, it is generally believed that the matter composition of clusters averaged over a sufficiently large volume should be representative of the universe. Second, present X-ray observations have revealed an increase in the baryon fraction of clusters with radii and no evidence for an asymptotic tendency toward a universal value at large radii (White \& Fabian 1995; David 1997; White, Jones, \& Forman 1997; Ettori \& Fabian 1999; Jones \& Forman 1999; Markevitch et al. 1999). This conflict has probably arisen from the oversimplification of the currently adopted temperature model for the intracluster gas. Setting a priori the universality of $f_{b}\left(r_{\text {vir }}\right)$ can instead allow us to work out the constraints on the radial variations of the density and temperature of the gas. Third, the boundary condition will be required mathematically in order to solve the hydrostatic equation, while little has been known about the gas properties within the central cores of clusters, where local dynamical activities may play a dominant role.

In a word, if the distribution of dark matter can be reasonably approximated by the NFW profile, we will be able to derive the radial density and temperature profiles of intracluster gas based on the well-measured X-ray surface brightness of clusters with the constraint that the baryon fraction within the virial radius is universal. The resulting density and temperature profiles can be directly compared with the X-ray spectroscopic measurements and the SZ observations of clusters, which will provide a critical test for the validity of the NFW profile and the hydrostatic equilibrium hypothesis. Meanwhile, such a study will be helpful for resolving the conflict regarding the different temperature measurements, i.e., whether or not the X-ray-emitting gas can be regarded as isothermal in clusters. Eventually, we hope that this research will be of significance for our understanding of the fundamental physical processes in the dynamical evolution of clusters, including nongravitational heating.

\section{THE MODEL}

We assume that the dark halo of a cluster is described by the NFW profile,

$$
\rho_{\mathrm{DM}}(r)=\frac{\rho_{s}}{\left(r / r_{s}\right)\left(1+r / r_{s}\right)^{2}},
$$

where $\rho_{s}$ and $r_{s}$ are the characteristic density and length, respectively. Another two equivalent parameters frequently used in the literature are $\delta_{c} \equiv \rho_{s} / \rho_{\text {crit }}$ and $c \equiv r_{\text {vir }} / r_{s}$. The virial radius, $r_{\mathrm{vir}}$, is defined by

$$
M_{\mathrm{DM}}\left(r_{\mathrm{vir}}\right)=\frac{4 \pi}{3} r_{\mathrm{vir}}^{3} \Delta_{c} \rho_{\text {crit }},
$$

where $\Delta_{c}$ represents the overdensity of dark matter with respect to the average background value $\rho_{\text {crit }}$, for which we take $\Delta_{c}=200$ in the following computation. Our main conclusion is unaffected by this choice. The total mass of the dark matter halo enclosed within radius $r$ is

$$
M_{\mathrm{DM}}(r)=4 \pi \rho_{s} r_{s}^{3}\left[\ln \left(1+\frac{r}{r_{s}}\right)-\frac{r}{r+r_{s}}\right] .
$$

On the other hand, the X-ray surface brightness profile, $S_{\mathrm{X}}(r)$, can be approximated by the conventional $\beta$ model,

$$
S_{\mathrm{X}}(r)=S_{0}\left(1+\frac{r^{2}}{r_{c}^{2}}\right)^{-3 \beta+1 / 2},
$$

where $r_{c}$ denotes the core radius. For the thermal bremsstrahlung emission, the above form of $S_{\mathrm{X}}(r)$ indicates (Cowie, Henriksen, \& Mushotzky 1987)

$$
n_{\text {gas }}(r) T^{1 / 4}(r)=n_{\text {gas }, 0} T_{0}^{1 / 4}\left(1+\frac{r^{2}}{r_{c}^{2}}\right)^{-3 \beta / 2},
$$

where $n_{\text {gas }}$ and $T$ are the gas number density and temperature, respectively. Equation (5) identifies the $\beta$ density profile in the case of isothermality. As a result, the total mass in gas within $r$ is simply

$$
M_{\mathrm{gas}}(r)=4 \pi \mu m_{p} n_{\mathrm{gas}, 0} \int\left(\frac{T_{0}}{T}\right)^{1 / 4}\left(1+\frac{r^{2}}{r_{c}^{2}}\right)^{-3 \beta / 2} r^{2} d r,
$$

where $\mu=0.585$ is the average molecular weight. The volume-averaged baryon fraction, $f_{b}(r)$, within $r$ is defined as

$$
f_{b}(r)=\frac{M_{\mathrm{gas}}(r)}{M_{\mathrm{gas}}(r)+M_{\mathrm{DM}}(r)} .
$$

Here we have neglected the contribution of stellar mass to $f_{b}(r)$. We further assume that the gas is in hydrostatic equilibrium with the underlying gravitational potential of the cluster produced by $M_{\mathrm{tot}}(r) \equiv M_{\mathrm{gas}}(r)+M_{\mathrm{DM}}(r)$, i.e.,

$$
\frac{G\left[M_{\mathrm{gas}}(r)+M_{\mathrm{DM}}(r)\right]}{r^{2}}=-\frac{1}{\mu m_{p} n_{\mathrm{gas}}} \frac{d\left(n_{\mathrm{gas}} k T\right)}{d r} .
$$

Using the temperature and baryon fraction as the two variables and reorganizing the above equations yields

$$
\begin{aligned}
& \quad \frac{d \tilde{T}}{d x}=\frac{4 \beta x \widetilde{T}}{x^{2}+a^{2}}-\frac{4 \alpha_{0}}{3 x^{2}}\left[\ln (1+x)-\frac{x}{1+x}\right] \frac{1}{1-f_{b}}, \\
& \frac{d f_{b}}{d x}= \\
& \frac{\left(1-f_{b}\right)^{2} b \tilde{T}^{-1 / 4}\left[1+\left(x^{2} / a^{2}\right)\right]^{-3 \beta / 2} x^{2}-f_{b}\left(1-f_{b}\right)\left[x /(1+x)^{2}\right]}{\ln (1+x)-x /(1+x)},
\end{aligned}
$$

where the scaled quantities are $\tilde{T}=T / T_{0}, x=r / r_{s}, a=$ $r_{c} / r_{s}, b=\mu m_{p} n_{\text {gas }, 0} / \rho_{s}$, and $\alpha_{0}=4 \pi G \mu m_{p} \rho_{s} r_{s}^{2} / k T_{0}$. Our task is thus reduced to finding the solutions of equations (9) and (10) with the boundary conditions

$$
\widetilde{T}(0)=1
$$

and

$$
\begin{aligned}
& f_{b}(c)=f_{b, \mathrm{BBN}} \\
& \left.\frac{d f_{b}}{d x}\right|_{x=c}=0 .
\end{aligned}
$$

This last constraint demands that the baryon fraction should asymptotically match the universal value of $f_{b, \mathrm{BBN}}$ at the virial radius $r_{\text {vir }}$, or equivalently $c$.

\section{RESULTS}

\subsection{General Properties}

There are a total of five parameters involved in equations (9) and (10): $\beta, \alpha_{0}, a, b$, and $\delta_{c}$ or $c$. In addition, the universal 
baryon fraction $f_{b \text {, BBN }}$ should also be regarded as another unknown parameter, although $\Omega_{b}$ has been fixed by big bang nucleosynthesis. However, these parameters are not independent, as we discuss in some detail later. Mathematically, the number of these free parameters can be reduced by two with the restriction of the two first-order differential equations, equations (9) and (10), and the associated boundary conditions. Namely, given four of the six parameters $(\beta$, $\left.\alpha_{0}, a, b, \delta_{c}, f_{b, \text { BBN }}\right)$, we will be able to find the other two from equations (9) and (10). Technically, we iteratively perform the numerical searches for the solutions of equations (9) and (10) over a space of two parameters until the boundary condition equations (11)-(13) are satisfied. Using the resulting temperature $\tilde{T}$, we then derive the scaled gas density $\tilde{n}_{\text {gas }} \equiv n_{\text {gas }}(r) / n_{\text {gas }, 0}$ according to equation (5):

$$
\tilde{n}_{\text {gas }}=\tilde{T}^{-1 / 4}\left(1+\frac{r^{2}}{r_{c}^{2}}\right)^{-3 \beta / 2}
$$

We demonstrate in Figure 1 a set of typical solutions for $a=0.3, \delta_{c}=10^{4}$, and $f_{b, \text { BBN }}=0.1$, but with different choices of $\beta: \frac{2}{3}, 0.75,0.85$, and 1 . The other two parameters, $b$ and $\alpha_{0}$, can be uniquely determined in each case during our numerical searches for the solutions, which read $\left(b, \alpha_{0}\right)=$ $(0.34,5.73),(0.42,6.22),(0.65,6.61)$, and $(1.34,6.96)$ for $\beta=\frac{2}{3}$, $0.75,0.85$, and 1 , respectively. The halo concentration for our example is found to be $c=r_{\text {vir }} / r_{s}=5.32$. We have also displayed in Figure 1 the input $S_{\mathrm{X}}(r)$ and our derived radial profiles of gas density, $\tilde{n}_{\text {gas }}$.

It is not surprising from Figure 1 that the shape of the resulting radial profiles of gas density, $\tilde{n}_{\text {gas }}$, are very similar to the conventional expectation, i.e., the $\beta$ model. Alternatively, the baryon fraction, $f_{b}(r)$, shows an increase with radius roughly within the core regions, and then asymptotically approaches the background value $f_{b, \mathrm{BBN}}$ at virial radius $c$ (for a small $\beta$ ) or reaches a maximum before it matches the background value (for a large $\beta$ ). However, the temperature profile $\tilde{T}(r)$ derived from equations (9) and (10) along with the restrictions equations (11)-(13) exhibits a remarkable difference in the radial variation among different $\beta$ clusters, from a rapid increase at large radii for a small $\beta$ to a sharp drop near the virial radius for a large $\beta$, separated roughly at $\beta \approx 0.8$. This property is mainly due to our restriction on the baryon fraction at $x=c$ (eqs. [12] and [13]): $f_{b}=f_{b, \text { BBN }}$ and $d f_{b} / d x=0$. Recall that a similar temperature behavior is required in order for $f_{b}$ to asymptotically approach the universal value $f_{b, \mathrm{BBN}}$ at large radii within the framework of the $\beta$ model and the hydrostatic equilibrium for intracluster gas, in which the separation of the two different variations of the asymptotic temperature at large radii occurs at $\beta=\frac{2}{3}$ (Wu \& Xue 2000b).

Similarly, we can numerically obtain the eigenvalues $\left(\alpha_{0}\right.$, $b)$ for different choices of $\left(\beta, a, \delta_{c}, f_{b, \mathrm{BBN}}\right)$. In Figure 2 we display the dependence of the resulting eigenvalues $\left(\alpha_{0}, b\right)$ on each of the four parameters. The most interesting result is the cutoff of the $\beta$ parameter set by $\alpha_{0}=0$, below which unphysical solutions will occur. This indicates that the observed X-ray surface brightness profile $S_{\mathrm{X}}(r)$ described by the $\beta$ model should not have too-small values of $\beta$ if our working scenario holds true. In order to demonstrate how

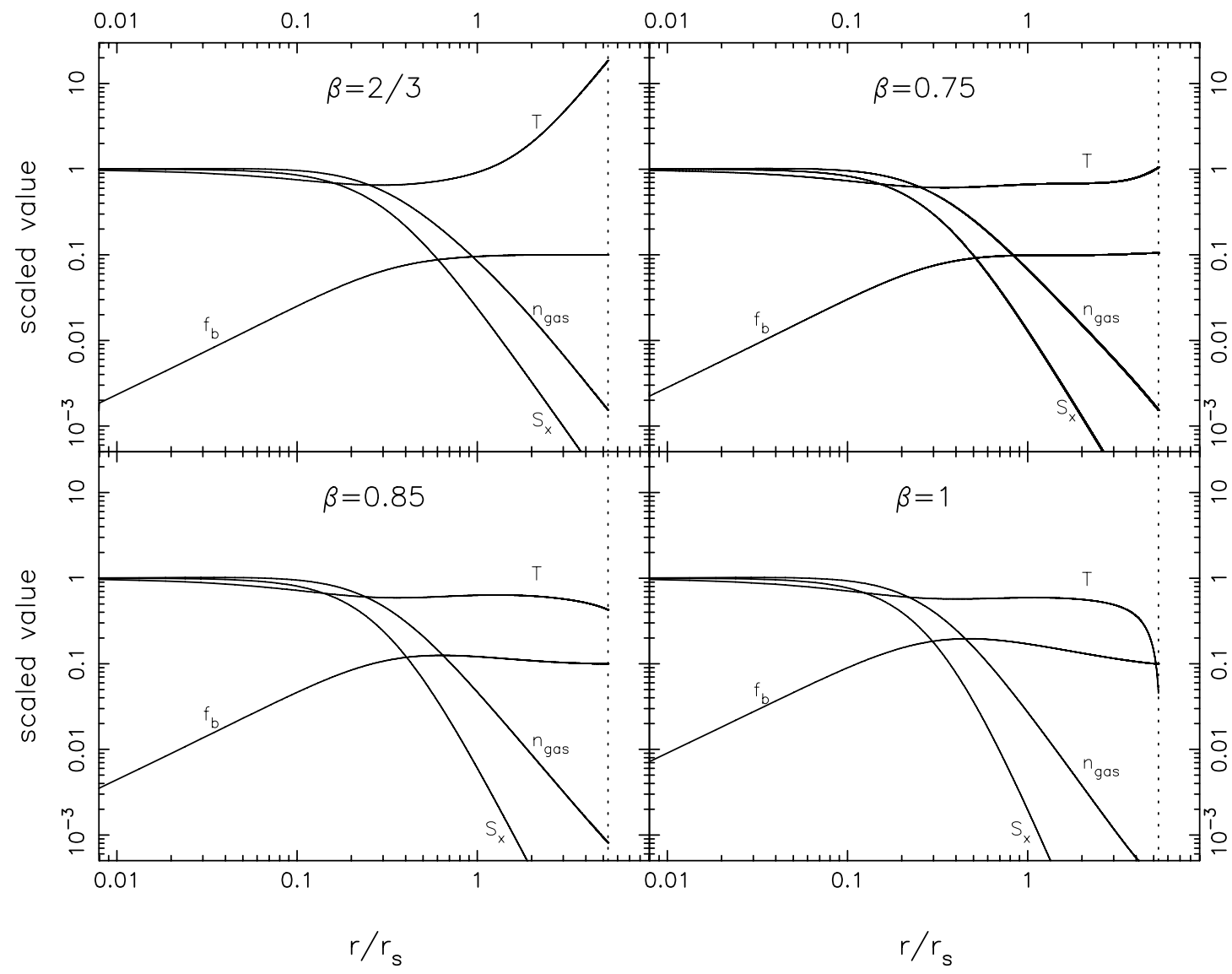

FIG. 1.-Derived radial profiles of gas density $\left(n_{\text {gas }} / n_{\text {gas }, 0}\right)$, temperature $\left(T / T_{0}\right)$, and baryon fraction $\left(f_{b}\right)$ for $f_{b, \mathrm{BBN}}=0.1, a=r_{c} / r_{s}=0.3$, and $\delta_{c}=\rho_{s} / \rho_{\text {crit }}=$ $10^{4}$, but with four different choices of $\beta$. Also shown are the input X-ray surface brightness profiles of clusters described by the conventional $\beta$ model. The dotted line denotes the virial radius. 


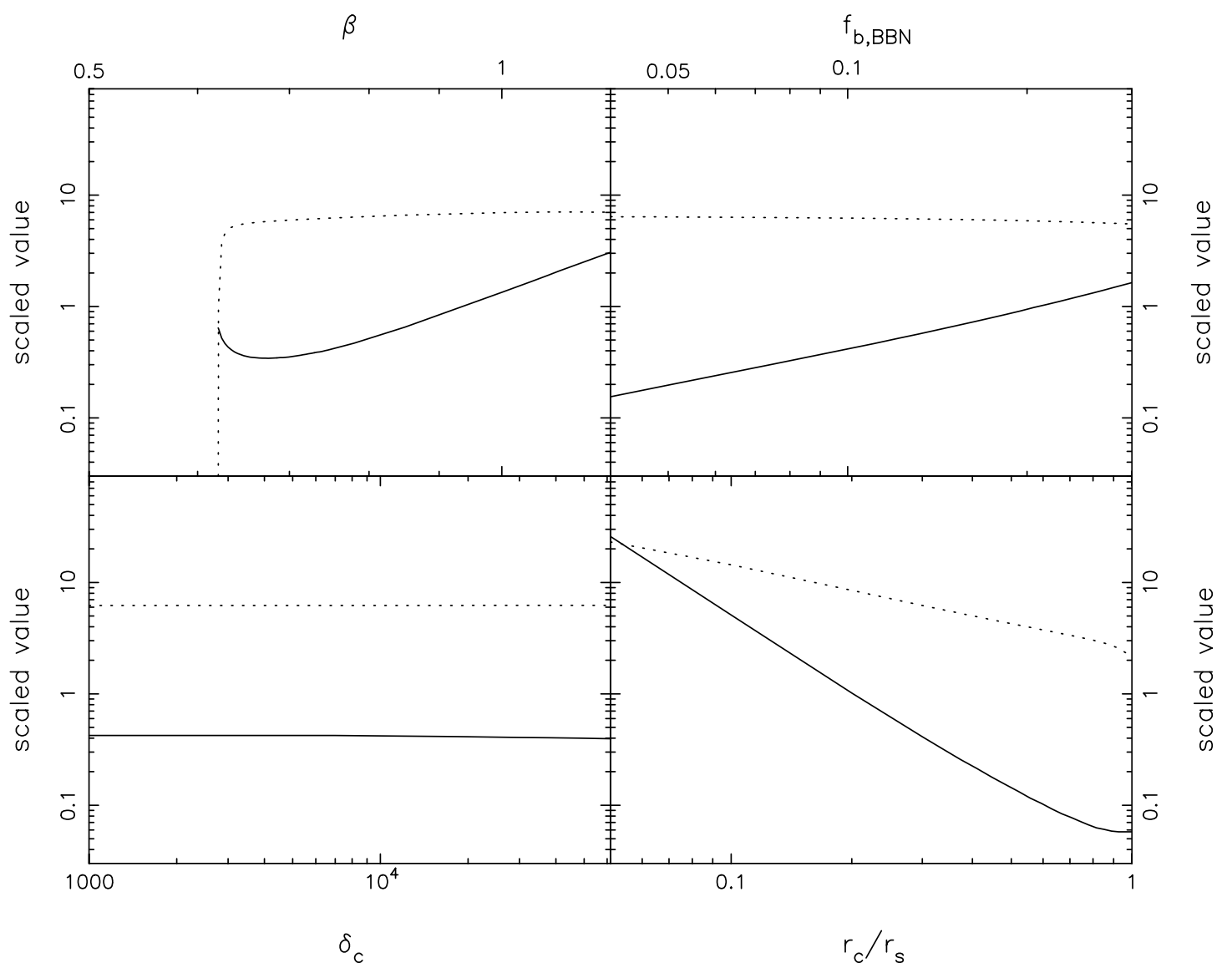

FIG. 2.-Dependence of $\alpha_{0}\left(=4 \pi G \mu m_{p} \rho_{s} r_{s}^{2} / k T_{0}\right.$; dotted lines $)$ and $b\left(=\mu m_{p} n_{\text {gas }, 0} / \rho_{s} ;\right.$ solid lines $)$ on $\beta\left(\delta_{c}=10^{4}, a=0.3\right.$, and $f_{b, \mathrm{BBN}}=0.1$; top left $), f_{b, \mathrm{BBN}}$ $\left(\beta=0.75, \delta_{c}=10^{4}\right.$, and $a=0.3 ;$ top right $), \delta_{c}\left(\beta=0.75, a=0.3\right.$, and $f_{b, \mathrm{BBN}}=0.1 ;$ bottom left $)$, and $a\left(\beta=0.75, \delta_{c}=10^{4}\right.$, and $f_{b, \mathrm{BBN}}=0.1 ;$ bottom right $)$.

the cutoff value, $\beta_{\text {cut }}$, varies with clusters, we perform a search for the solutions with $\alpha_{0}=0$. In this case, the eigenvalue $b$ can be uniquely fixed by our numerical solutions. Meanwhile, our numerical search shows that $f_{b \text {, BBN }}$ produces a minor effect on $\beta_{\text {cut }}$. Consequently, $\beta_{\text {cut }}$ depends mainly on two parameters: $\delta_{c}$ and $a$ (Fig. 3). It turns out that for a typical cluster with $\delta_{c}=10^{3}-10^{5}$ and $a=0.1-1$, the $\beta$ parameter should be greater than 0.5 , and $\beta_{\text {cut }}$ may exceed 0.6 if $\delta_{c}>10^{5}$. These constraints on the $\beta$ parameter seem to be in good agreement with the X-ray observations that the majority of the X-ray clusters have X-ray surface brightness profiles that satisfy $\beta>0.5$.

Essentially, the parameter $\alpha_{0}$ depends very weakly on $\delta_{c}$, $f_{b, \mathrm{BBN}}$, and $\beta$ (for $\beta>\beta_{\text {cut }}$ ), and its typical values for $a=0.15$ and 0.3 are around 10 and 6.5 , respectively. Our estimates can be compared with previous studies that assumed an isothermal gas distribution tracing the gravitational potential of the NFW profile, but dropping the restrictions of equations (12)-(13): for rich clusters, the variation of $\alpha_{0}$ is limited to a very narrow range from 6 to 25 , with an average value of $\sim 10$ (Ettori \& Fabian 1999; Wu \& Xue 2000a; Wu 2000). Actually, $\alpha_{0}$ can be approximately regarded as a constant for a given $a$ (see Fig. 2).

\subsection{Asymptotic Temperature at Large Radii}

We learn from Figure 1 that there exists a critical value of $\beta_{\text {crit }}$ such that $\tilde{T}(c)=1$. Namely, the radial temperature profile close to $x=c$ will show a tendency to increase with radius when $\beta<\beta_{\text {crit }}$ (and decrease when $\beta>\beta_{\text {crit }}$ ). The existence of such a $\beta_{\text {crit }}$ can be understood qualitatively by considering the following limiting situation, in which the virial radius $c$ is much greater than the gas core radius $a$.

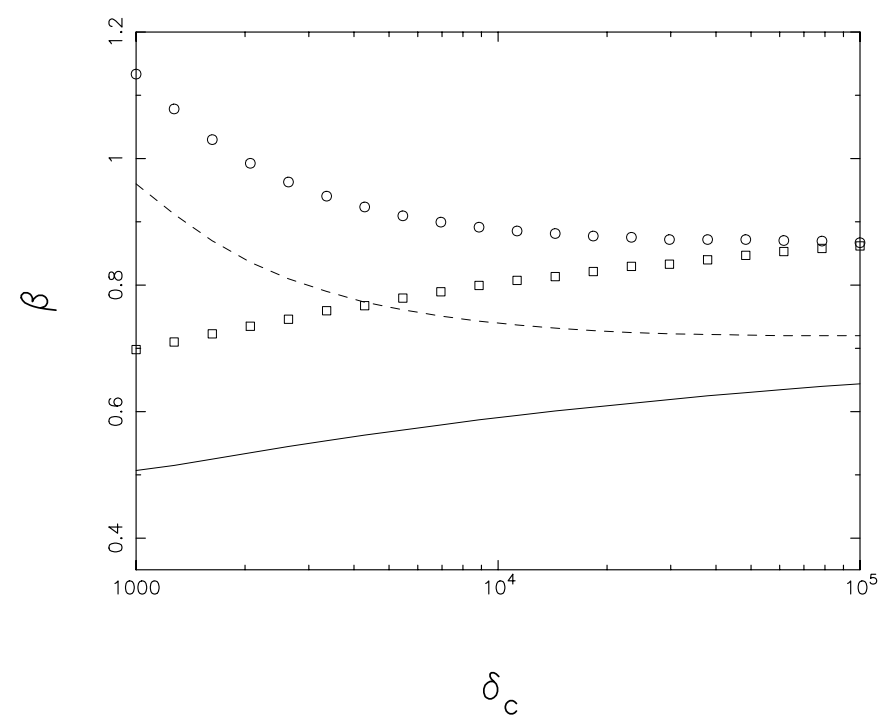

FIG. 3.-Lower limits on $\beta$ parameter against the characteristic density $\delta_{c}$ for two choices of $a:$ (1) $a=0.1$ (solid line and open squares) and (2) $a=1$ (dashed line and open circles). Solid and dashed lines: the limits $\beta_{\text {cut }}$ from the unphysical solutions of eqs. (9) and (10) as a result of $\alpha_{0}=0$; open squares and circles: limits $\beta_{\text {crit }}$ set by the requirement $T(c) / T_{0}=1$. 
TABLE 1

$\delta_{c}$ AND $r_{s}$ FOR A TYPICAL ClUSTER

\begin{tabular}{|c|c|c|c|c|}
\hline$\beta$ & $\begin{array}{c}r_{s} \\
(\mathrm{Mpc})\end{array}$ & $\delta_{c}$ & $c$ & $\widetilde{T}(c)$ \\
\hline $0.70 \ldots \ldots$ & 0.51 & 7051 & 4.54 & 4.66 \\
\hline $0.75 \ldots \ldots$ & 0.49 & 7314 & 4.63 & 2.56 \\
\hline $0.80 \ldots \ldots$ & 0.41 & 8068 & 4.84 & 2.00 \\
\hline $0.85 \ldots \ldots$ & 0.39 & 8600 & 4.98 & 0.77 \\
\hline $1.00 \ldots \ldots$ & 0.29 & 11000 & 5.54 & 0.06 \\
\hline
\end{tabular}

Note.-For a typical cluster with $T_{0}=7 \mathrm{keV}$, $r_{c}=0.25 \mathrm{Mpc}$, and $n_{\mathrm{gas}, 0}=5 \times 10^{-3} \mathrm{~cm}^{-3}$.

The universal profile admits an $x^{-3}$ density at large $x$. If the distribution of $f_{b, \mathrm{BBN}}$ is to be asymptotically flat, the gas density $\tilde{n}_{\text {gas }}$ must also obey $x^{-3}$ at large $x$. Given that the $\beta$ model requires equation (14) to hold, the existence of a $\beta_{\text {crit }}$ thus naturally follows. For this limiting situation $\beta_{\text {crit }}=1$, but when $c$ no longer significantly exceeds $a$, the value of $\beta_{\text {crit }}$ is found to vary somewhat, as described below.

While the asymptotic high and low temperature are both allowed mathematically, there should be one temperature profile at large cluster radii. Indeed, it is very unlikely that the gas temperature would increase monotonically with outward radius. At least, there is no known mechanism that can produce a significant rise of gas temperature at the outermost radii. Note that this does not exclude a mildly increasing temperature with radius over a certain region of clusters (e.g., Irwin \& Bregman 2000). As a result, the condition $\widetilde{T}(c)=1$ sets another low limit on the value of $\beta$, which guarantees that the gas temperature would not rise at the virial radius when $\beta>\beta_{\text {crit }}$.

In order to determine $\beta_{\text {crit }}$, we once again perform numerical searches for the solutions of equations (9) and (10), with the additional constraint $\widetilde{T}(c)=1$. This last point reduces the free parameters to three: $f_{b, \mathrm{BBN}}, \delta_{c}$, and $a$. In Figure 3 we plot the resulting $\beta_{\text {crit }}$ against $\delta_{c}$ for two different choices of $a(a=0.1$ and 1$)$ but a fixed universal baryon fraction of $f_{b, \mathrm{BBN}}=0.1$. It appears that although $\beta_{\text {crit }}$ shows a radial variation similar to that of $\beta_{\text {cut }}$, the value of $\beta_{\text {crit }}$ is apparently larger than the corresponding result for $\beta_{\text {cut }}$, indicating that the observed X-ray surface brightness profiles of clusters should possess an even larger $\beta$ parameter than $\beta_{\text {cut }}$ to ensure the natural drop of gas temperature at the edges of clusters. For our examples shown in Figure 3, the $\beta$ parameters are required to exceed 0.7 and 0.8 for $\delta_{c}=10^{3}$ and $10^{4}$, respectively. This may constitute a critical test for the working hypotheses established in the above section. The currently available data from X-ray measurements seem to marginally agree with these limits even if the effect of cooling flows (e.g., Vikhlinin, Forman, \& Jones $1999)$ and the unknown parameters $\left(a, \delta_{c}\right.$, and $\left.f_{b, \mathrm{BBN}}\right)$ are taken into account. We explore the possible reasons for this in detail in $\S 4$.

\subsection{Self-Consistent Solution}

In an optically thin plasma emission model, the central gas density, $n_{\text {gas }, 0}$, is related to the central surface brightness, $S_{\mathrm{X}}(0)$, through

$$
n_{\mathrm{gas}, 0}^{2}=4 \pi^{1 / 2} \frac{1}{\bar{g} \bar{\mu}} \frac{1}{\alpha\left(T_{0}\right)} \frac{\Gamma(3 \beta)}{\Gamma(3 \beta-1 / 2)} \frac{S_{\mathrm{X}}(0)}{r_{c}},
$$

where $\alpha\left(T_{0}\right)$ is the cooling function

$$
\alpha\left(T_{0}\right)=\left(\frac{2^{4} e^{6}}{3 m_{\mathrm{e}} \hbar c^{2}}\right)\left(\frac{2 \pi k T_{0}}{3 m_{\mathrm{e}} c^{2}}\right)^{1 / 2},
$$

$\bar{g}$ is the average Gaunt factor, and $\bar{\mu} \equiv \mu^{2}(1+X) / 2$, where $X=0.768$ is the primordial hydrogen mass fraction. The $\mathrm{X}$-ray measurement of the surface brightness profile of a cluster provides us with the quantities $S_{\mathrm{X}}(0), \beta$, and $r_{c}$. On the other hand, the central electron temperature, $T_{0}$, can be obtained relatively easily by X-ray spectroscopic measurements as compared to the outermost temperature, e.g., $T(x \approx c)$. Given $S_{\mathrm{X}}(0), \beta, r_{c}$, and $T_{0}$, we will be able to derive the central gas density $n_{\mathrm{gas}, 0}$ in terms of equations (15) and (16). Moreover, since the purpose of the present paper is not to estimate the baryon fraction of clusters, we assume that the universal value of $f_{b, \text { BBN }}$ is known. As a result, there are only two parameters, $\rho_{s}$ (or equivalently $\delta_{c}$ ) and $r_{s}$ in the NFW profile, that remain to be determined. We thus search for the solutions of equations (9) and (10) by iterations with a set of $\left(\delta_{c}, a\right)$ until a self-consistent solution is achieved, which uniquely determines the parameters $\delta_{c}$ and $r_{s}$ in the NFW profile. Recall that in the isothermal case, the X-ray surface brightness profile predicted by the NFW profile is directly applicable to the fitting of the observed $S_{\mathrm{X}}(r)$, giving rise to the two parameters $\delta_{c}$ and $r_{s}$ (Makino et al. 1998; Ettori \& Fabian 1999; Wu \& Xue 2000a, 2000c; Wu 2000). Indeed, it appears that the procedure for finding the two parameters in the NFW profile now becomes very complicated if the temperature profile of a cluster is unknown.

In the present paper we do not intend to determine the parameters $\left(\rho_{s}, r_{s}\right)$ by applying the current method to an ensemble of X-ray clusters. Instead, we present the result for a typical cluster with $n_{\mathrm{gas}, 0}=5 \times 10^{-3} \mathrm{~cm}^{-3}, r_{c}=0.25$ $\operatorname{Mpc}\left(H_{0}=50 \mathrm{~km} \mathrm{~s}^{-1} \mathrm{Mpc}^{-1}\right)$, and $T_{0}=7 \mathrm{keV}$, but different $\beta$ parameters. We fix the universal baryon fraction to be $f_{b, \mathrm{BBN}}=0.1$. The resulting $\rho_{s}$ and $r_{s}$, along with the concentration parameter $c$ (or equivalently, the virial radius) and the corresponding temperature $\widetilde{T}(c)$ at $x=c$, are listed in Table 1. Apart from the significant differences in the outermost temperatures at virial radii for different $\beta$ parameters, the values of the remaining parameters, $r_{s}, \delta_{c}$, and $c$, all fall inside the limits for typical rich clusters with an isothermal gas distribution (Ettori \& Fabian 1999; Wu \& Xue 2000a, 2000c; Wu 2000). This implies, on the other hand, that the determination of the two parameters in the NFW profile from the X-ray observations of intracluster gas is probably insensitive to the radial temperature variations. However, the temperature rise or drop near the edge of a cluster depends critically on the $\beta$ parameter.

\section{DISCUSSION AND CONCLUSIONS}

The current X-ray measurements of the radial temperature profiles of intracluster gas, especially at outer radii, have some ambiguity, which is probably responsible for the major uncertainties in the present determination of the gravitating masses of clusters. In addition, the large dispersion of the derived baryon fraction among different clusters may be associated with the poorly constrained cluster temperatures. Consequently, the cosmological application of the dynamical properties of clusters is significantly affected. In the absence of a reliable constraint on the radial temperature variation from X-ray spectral measurements, we have made an attempt in the present paper to derive the 
temperature profile $T(r)$ based on well-established facts from the X-ray observations and numerical simulations, along with some plausible boundary conditions. Specifically, we use the NFW profile as the dark matter distribution $n_{\mathrm{DM}}(r)$ of clusters, and assume that intracluster gas $n_{\mathrm{gas}}(r)$ is in hydrostatic equilibrium with the underlying gravitational potential dominated by $n_{\mathrm{DM}}(r)$ and $n_{\text {gas }}(r)$. We adopt the X-ray observed surface brightness profile $S_{\mathrm{X}}(r)$ described by the conventional $\beta$ model to set up a link between gas density, $n_{\text {gas }}(r)$, and temperature, $T(r)$. We have then numerically solved the hydrostatic equation by demanding that the baryon fraction $f_{b}(r)$ asymptotically approaches the universal value $f_{b, \mathrm{BBN}}=\Omega_{b} / \Omega_{m}$ at the viral radius. As a result, we can simultaneously determine the radial profiles of gas density, temperature, and baryon fraction in clusters. In particular, using the X-ray data of $\beta, r_{c}$, $S_{\mathrm{X}}(0)$, and $T_{0}$, we are able to find the two free parameters in the NFW profile, with which the dark matter profile can be completely fixed.

The temperature profile constructed jointly from the NFW profile and the X-ray surface brightness measurement appears to be significantly different from the conventional speculation. Apparently, our temperature profiles (see Fig. 1) cannot be simply described by the widely adopted polytropic equation of state, $T \propto n_{\text {gas }}^{\gamma-1}$, where $\gamma$ is the polytropic index, which is reflected by the striking difference between the core radius of gas density and that of temperature. It seems that the shapes of temperature profiles in the outer regions of clusters can be dramatically different, depending sensitively on the $\beta$ parameter. This point has mainly arisen from the restriction that the volume-averaged baryon fraction should asymptotically approach the universal value at the virial radius. The recent study by Wu \& Xue (2000b) based on the $\beta$ model and the polytropic gas distribution has essentially reached a similar result, i.e., a mild increase in temperature with radius should be required for clusters with $\frac{1}{3}<\beta<\frac{2}{3}$ in order to ensure the universal constancy of the cluster baryon fraction at large radii. Although this possibility has been reported for some clusters by Irwin \& Bregman (2000) and is shown in Figure 1 for small $\beta$ (more clearly in Fig. 4 for $\beta=\frac{2}{3}$ ), naturally we would expect a drop in temperature at the outermost radii. This can be achieved if the $\beta$ parameters become larger (typically $\beta>0.8$ ). While numerical studies of cluster formation and evolution often yield a similar result of $\beta \approx 0.7-1$, observationally, it is unlikely that one can significantly raise the $\beta$ value to $\beta>0.8$ from a fitting of the X-ray surface brightness profiles of clusters. Exclusion of the central cooling regions or employment of the double- $\beta$ models in fitting the observed $S_{\mathrm{X}}(r)$ can only lead to a moderate increase of the $\beta$ parameter (e.g., Jones \& Forman 1984; Vikhlinin et al. 1999; Xue \& Wu 2000a).

In addition to the X-ray spectroscopic measurements, the SZ effect may also help to make a distinction between the temperature rise or fall models at large radii close to $r_{\text {vir }}$. The SZ effect depends on the gas density and temperature via $\Delta T / T_{\mathrm{CBR}} \propto \int n_{\mathrm{gas}} T d l$, where $T_{\mathrm{CBR}}$ represents the temperature of the cosmic background radiation, and the integral is performed along the line of sight $l$. In Figure 4 we demonstrate the thermal SZ effect produced by two clusters with $\beta=\frac{2}{3}$ and 1 , respectively, adopting the same parameters as in Figure 1. If the gas temperature shows a dramatic increase with radius, the shape of the SZ profile will be strongly distorted. On the contrary, it turns out to be diffi-

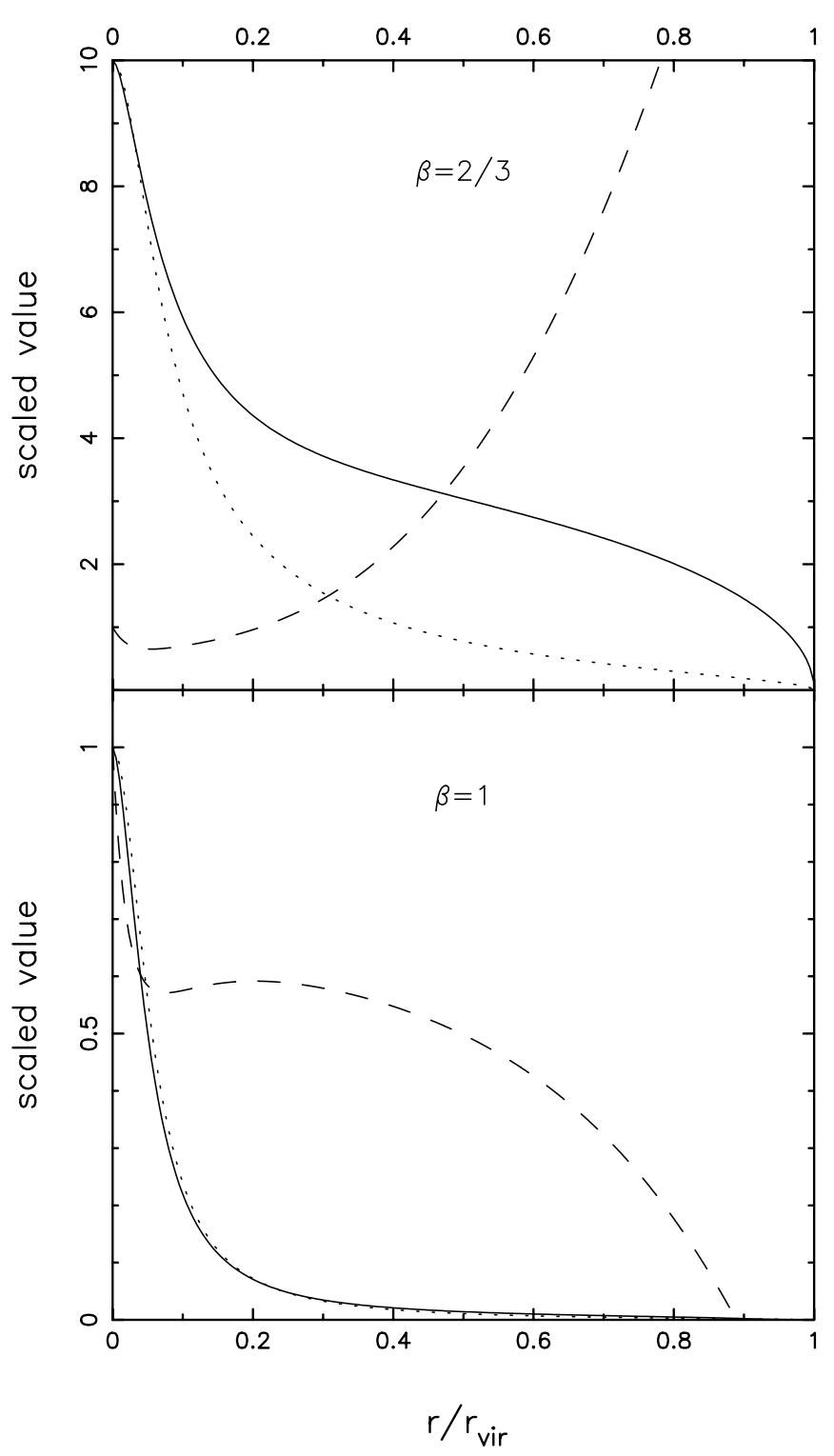

FIG. 4.-Comparison of the SZ profiles predicted by the isothermal gas distribution (dotted lines) and our derived radial distribution of intracluster gas from eqs. (9) and (10) (solid lines). Top and bottom panels correspond to the situations of $\beta=\frac{2}{3}$ and $\beta=1$ in Fig. 1, respectively. The temperature profiles are shown by dashed lines. For clarity, the vertical scales for $\beta=\frac{2}{3}$ (top) have been multiplied by a factor of 10 .

cult to distinguish the temperature-decreasing model from the isothermal one simply based on the $\mathrm{SZ}$ observations.

Although we have fulfilled our task that the radial profiles of the density and temperature of intracluster gas are derived from well-established facts, the existing X-ray data and future X-ray observations can provide a critical test of these predictions. Any conflict between observations and our predictions would imply that at least one of the following working hypotheses should be abandoned:(1) the NFW profile as the dark matter distribution of clusters; (2) the hydrostatic equilibrium for intracluster gas; (3) pure thermal bremsstrahlung as the observed X-ray emission; or (4) the total baryon fraction of clusters as a reliable indicator of the matter mixture of the universe. In fact, all these hypotheses have been challenged in recent years.

It has been noticed that the central cusp in the NFW profile disagrees with the soft inner mass distributions of 
galaxies and clusters, which has led Spergel \& Steinhardt (2000) to propose the possibility that the cold dark matter particles are self-interacting. Indeed, the X-ray surface brightness of clusters predicted from the NFW profile has a much smaller core radius than the one seen from X-ray observations (Makino et al. 1998). In fact, the cusped NFW profile yields a higher central gas temperature, which can be clearly seen from the temperature profiles plotted linearly in Figure 4, while the X-ray spectroscopic measurements often reveal a cold gas component inside the core regions. As a result, a modification to the NFW profile should be properly made, such as the empirical density profile with a definite core radius $\rho_{\mathrm{DM}}=\rho_{0}(1+x)^{-1}\left(1+x^{2}\right)^{-1}$ (Burkert 1995), which may both provide a good fit to the dark matter distribution and predict the correct shape of the X-ray surface brightness distribution of clusters $(\mathrm{Wu} \&$ Xue 2000c). However, fitting the predicted X-ray surface brightness profiles by such a revised dark halo profile to an ensemble of the X-ray observed surface brightness profiles of clusters, Wu \& Xue (2000c) have shown that the core radii of the dark matter halos reach the X-ray core sizes of $\sim 0.2 \mathrm{Mpc}$. The recent numerical simulations under some simple models for self-interacting dark matter particles have essentially arrived at the similar conclusion (Yoshida et al. 2000). Such large dark matter cores apparently exceed the ones required by modeling giant arcs seen in the central regions of clusters (Hammer 1991; Wu \& Hammer 1993; Grossman \& Saha 1994; Tyson et al. 1998). In a sense, any attempts at significantly modifying the NFW profile may require novel physical mechanisms.

Alternatively, it has been debated in recent years whether the intracluster gas is in perfect hydrostatic equilibrium with the underlying gravitational potential, based mainly on the presence of substructures and complex temperature patterns in clusters revealed by optical/X-ray observations (e.g., Henriksen \& White 1996; Markevitch 1996). Yet, a statistical comparison of cluster masses derived from optical/X-ray observations and the NFW profile, under the assumption of hydrostatic equilibrium, and the strong/weak gravitational lensing measurements shows excellent agreement among different cluster mass estimates on scales greater than the X-ray core radii (Allen 1998; Wu et al. 1998; Wu 2000, and references therein). This suggests that hydrostatic equilibrium may break down only on small scales where the local dynamical activities make a nonnegligible contribution to cluster mass estimates, and hydrostatic equilibrium can be reasonably applicable to the overall distribution of intracluster gas.

Our emphasis is thus laid on the effect of nongravitational heating processes in the dynamical evolution of clusters. It has been discovered in past years that the observationally determined X-ray luminosity $\left(L_{\mathbf{X}}\right)$ and temperature relation, $L_{\mathrm{X}} \propto T^{3}$, deviates significantly from the prediction $L_{\mathrm{X}} \propto T^{2}$ under the standard scenario that assumes hydrostatic equilibrium and thermal bremsstrahlung emission for intracluster gas (e.g., David et al. 1993; Markevitch 1998). While one cannot definitely exclude the possibility that the baryon fraction of clusters may vary with temperature (David et al. 1993), this conflict is strongly suggestive of the importance of the nongravitational heating processes in the cosmic evolution of clusters. In fact, supernovae in cluster galaxies would substantially inject energy into the intracluster medium through galactic winds in the early phase of cluster formation, and the intracluster gas can be heated to temperatures even exceeding that produced by gravitational heating alone (White 1991; David, Forman, \& Jones 1991). This scenario may allow one to naturally reproduce the observed $L_{\mathrm{X}}-T$ relation $L_{\mathrm{X}} \propto T^{3}$ (Ponman, Cannon, \& Navarro 1999; Wu, Fabian, \& Nulsen 2000; Loewenstein 2000). In particular, the energy input from supernova-driven protogalactic winds will permit the intracluster gas to extend out to larger radii, giving rise to a shallower X-ray surface brightness profile (David et al. 1990; Ponman et al. 1999; Lloyd-Davies, Ponman, \& Cannon 2000). The discrepancy between the large $\beta$ parameters $(\beta>0.8)$ required to maintain the decreasing temperature profile near the virial radius discussed in the present paper and the relatively small value of $\beta \approx 0.7$ found from the X-ray observed surface brightness profiles of clusters is probably due to our negligence of the effects of other heating processes. A reliable construction of the radial density and temperature profiles of intracluster gas should also allow the nongravitational heating to be included.

Taking these arguments as a whole, we feel that the exercise made in this paper, combined with the X-ray observations of clusters, will be helpful for our understanding of the fundamental physical process that dominates the dynamical evolution of clusters. Applications of the current method to real X-ray clusters will be presented in subsequent work (Xue \& Wu 2000b).

We thank an anonymous referee for constructive comments that improved the presentation of this work. W. X. P. is grateful for the hospitality of the Department of Physics of the National Taiwan University, where part of this research was carried out. This work was supported by the National Science Foundation of China, under grant 1972531, and the National Science Council of Taiwan, under grant NSC 89-2112-M008-037.
Allen, S. 1998, MNRAS, 296, 392

Burkert, A. 1995, ApJ, 447, L25

Cole, S., \& Lacey, C. 1996, MNRAS, 281, 716

Cowie, L. L., Henriksen, M., \& Mushotzky, R. F. 1987, ApJ, 317, 593

David, L. P. 1997, ApJ, 484, L11

David, L. P., Arnaud, K. A., Forman, W., \& Jones, C. 1990, ApJ, 356, 32

David, L. P., Forman, W., \& Jones, C. 1991, ApJ, 380, 39

David, L. P., Slyz, A., Jones, C., Forman, W., \& Vrtilek, S. D. 1993, ApJ, 412,479

Eke, V. R., Navarro, J. F., \& Frenk, C. S. 1998, ApJ, 503, 569

Ettori, S., \& Fabian, A. C. 1999, MNRAS, 305, 834

Grossman, S. A., \& Saha, P. 1994, ApJ, 431, 74

Hammer, F. 1991, ApJ, 383, 66

Henriksen, M. J., \& White, R. E., III. 1996, ApJ, 465, 515

Irwin, J. A., \& Bregman, J. N. 2000, ApJ, 538, 543

Irwin, J. A., Bregman, J. N., \& Evrard, A. E. 1999, ApJ, 519, 518

\section{REFERENCES}

Jones, C., \& Forman, W. 1984, ApJ, 276, 38 1999, ApJ, 511, 65

Lloyd-Davies, E. J., Ponman, T. J., \& Cannon, D. B. 2000, MNRAS, 315, 689

Loewenstein, M. 2000, ApJ, 532, 17

Makino, N., Sasaki, S., \& Suto, Y. 1998, ApJ, 497, 555

Markevitch, M. 1996, ApJ, 465, L1

. 1998, ApJ, 503, 77

Markevitch, M., Forman, W. R., Sarazin, C. L., \& Vikhlinin, A. 1998, ApJ, 503,77

Markevitch, M., Vikhlinin, A., Forman, W. R., \& Sarazin, C. L. 1999, ApJ, 527,545

Navarro, J. F., Frenk, C. S., \& White, S. D. M. 1995, MNRAS, 275, 720 (NFW)

(N). 1997, ApJ, 490, 493

Ponman, T. J., Cannon, D. B., \& Navarro, J. F. 1999, Nature, 397, 135 
Silk, J., \& White, S. D. M. 1978, ApJ, 226, L103

Spergel, D. N., \& Steinhardt, P. J. 2000, Phys. Rev. Lett., 84, 3760

Suto, Y., Sasaki, S., \& Makino, N. 1998, ApJ, 509, 544

Tyson, J. A., Kochanski, G. P., \& Dell'Antonio, I. P. 1998, ApJ, 498, L107

Vikhlinin, A., Forman, W., \& Jones, C. 1999, ApJ, 525, 47

White, D. A. 2000, MNRÄS, 312, 663

White, D. A., \& Fabian, A. C. 1995, MNRAS, 273, 72

White, D. A., Jones, C., \& Forman, W. 1997, MNRAS, 292, 419

White, R. E., III. 1991, ApJ, 367, 69

Wu, K. K. S., Fabian, A. C., \& Nulsen, P. E. J. 2000, MNRAS, 318, 889

Wu, X.-P. 2000, MNRAS, 316, 299
Wu, X.-P., Chiueh, T., Fang L.-Z., \& Xue, Y.-J. 1998, MNRAS, 301, 861

Wu, X.-P., \& Hammer, F. 1993, MNRAS, 262, 187

Wu, X.-P., \& Xue, Y.-J. 2000a, ApJ, 529, L5 2000b, MNRAS, 311, 825 2000c, ApJ, 542, 578

Xue, Y.-J., \& Wu, X.-P. 2000a, MNRAS, 318, 715 2000 b, A\&A, 360, L43

Yoshida, N., Springel, V., White, S. D. M., \& Tormen, G. 2000, ApJ, 535, L103

Yoshikawa, K., \& Suto Y. 1999, ApJ, 513, 549 